\title{
Localisation Inverse Problem of Absorbing Laplacian Transport
}

\author{
Ibrahim Baydoun \\ École Centrale Paris, Laboratoire de MSS-MAT, Grande Voie des Vignes, \\ Châtenay-Malabry Cedex, France \\ Email: ibrahim.baydoun@ecp.fr
}

Received December 24, 2012; revised February 6, 2013; accepted March 7, 2013

Copyright (C) 2013 Ibrahim Baydoun. This is an open access article distributed under the Creative Commons Attribution License, which permits unrestricted use, distribution, and reproduction in any medium, provided the original work is properly cited.

\begin{abstract}
We study the localisation inverse problem corresponding to Laplacian transport of absorbing cell. Our main goal is to find sufficient Dirichelet-to-Neumann conditions insuring that this inverse problem is uniquely soluble. In this paper, we show that the conformal mapping technique is adopted to this type of problem in the two dimensional case.
\end{abstract}

Keywords: Laplacian Transport; Dirichlet-to-Neumann Operators; Inverse Problem; Conformal Mapping

\section{Introduction}

The theory of Dirichlet-to-Neumann operators is the basis of many research domains in analysis, particularly, those concerning Laplacian transports. It is also very important in mathematical-physics, geophysics, electrochemistry. Moreover, it is very useful in medical diagnosis, such as electrical impedance tomography, as showing in the following example:

Example 1. In 1989, J. Lee and G. Uhlmann have introduced an example on the determination of conductivity matrix field $\gamma(x)=\left[\gamma_{i, j}(x)\right]_{i, j=1}^{d}$, for $x$ in a bounded open domain $\Omega \subset \mathbb{R}^{d}$, see e.g. [1]. This example is related to measuring of elliptic Dirichlet-to-Neumann map for associated conductivity equation, see e.g. [1]. Notice that the solution of this problem has a lot of practical applications in various domains overall in medicine, which is an important diagnostic tool, e.g. in the electrical impedance tomography; the tissue in the human body is an example of highly anisotropic conductor [2].

Under assumption that there are no sources or sinks of current the potential $v(x), x \in \Omega$, for a given voltage $f(\omega), \omega \in \partial \Omega$, on the (smooth) boundary $\partial \Omega$ of $\Omega$ is a solution of the Dirichlet problem:

(P1) $\begin{cases}\operatorname{div}(\gamma \nabla v)=0 & \text { in } \Omega, \\ \left.v\right|_{\partial \Omega}=f & \text { on } \partial \Omega .\end{cases}$

Then the corresponding to (P1) Dirichlet-to-Neumann map (operator) $\Lambda_{\gamma, \partial \Omega}$ is (formally) defined by [3] as follows:

$$
\Lambda_{\gamma, \partial \Omega}:\left.f \mapsto \frac{\partial v_{f}}{\partial v_{\gamma}}\right|_{\partial \Omega}:=\left.v \cdot \gamma \nabla v_{f}\right|_{\partial \Omega} .
$$

Here $v$ is the unit outer-normal vector to the boundary at $\omega \in \partial \Omega$ and the function $v:=v_{f}$ is a solution of the Dirichlet problem (P1).

Dirichlet-to-Neumann operator (1.1) is also called the voltage-to-current map, since the function $\Lambda_{\gamma, \partial \Omega} f$ gives the induced current flux trough the boundary $\partial \Omega$. The key (inverse) problem is whether on can determine the conductivity matrix $\gamma$ by knowing electrical boundary measurements, i.e. the corresponding Dirichlet-to-Neumann operator? In general this operator does not determine the matrix $\gamma$ uniquely, see e.g. [4].

The main question in this context is to find sufficient conditions insuring that the inverse problem is uniquely soluble.

The problem of electrical current flux in the form (P1) is an example of so-called diffusive Laplacian transport. Besides the voltage-to-current problem, the motivation to study this kind of transport comes for instance, from the transfer across biological membranes, see e.g. [5,6]:

Example 2. Let some species of concentration $C(x), x \in \mathbb{R}^{d}$, diffuse stationary in the isotropic bulk $(\gamma=I)$ from a (distant) source localised on the closed boundary $\partial \Omega$ towards a semipermeable compact interface $\partial B$ of cell $\bar{B} \subset \Omega$, where they disappear at a given rate $W \geq 0$. Then the steady field of concentrations (Laplacian transport with a diffusion coefficient 
$D \geq 0$ ) obeys the set of equations:

(P2)

$$
\left\{\begin{array}{l}
\Delta C=0, x \in \Omega \backslash \bar{B}, \\
\left.C\right|_{\partial \Omega}\left(\omega_{0}\right)=f\left(\omega_{0}\right), \\
\text { the concentration at the source } \partial \Omega, \\
-\left.D \partial_{\nu} C\right|_{\partial B}(\omega)=\left.W\left(C-C^{*}\right)\right|_{\partial B}(\omega), \\
\text { on the interface } \omega \in \partial B .
\end{array}\right.
$$

Here $c^{*} \geq 0$ is the constant concentration of the species inside cell $\bar{B}$.

Now, similar to (1.1), we can associate with the problem (P2) a Dirihlet-to-Neumann operator

$$
\Lambda_{I, \partial \Omega}: f \mapsto \partial_{v} u_{f}:=\left.v \cdot \nabla u_{f}\right|_{\partial \Omega},
$$

with domain $\operatorname{dom}\left(\Lambda_{I, \alpha \Omega}\right)$ belongs to a certain Sobolev space, see Section 2.

The advantage of this approach is that as soon as the operator (1.2) is defined, one can apply it to study the mixed boundary value problem (P2). This gives, in particularly, the value of the particle flux due to Laplacian transport across the membrane $\partial \Omega$. Moreover, the total current across the boundary $\partial \Omega$ can be defined (for given $f$ ) in term of Dirihlet-to-Neumann operator (1.2) as follows:

$$
J_{\partial \Omega}:=-D \int_{\partial \Omega} \mathrm{d} \sigma \Lambda_{\gamma=I, \partial \Omega} f,
$$

where $\mathrm{d} \sigma$ designed the differential element relative to $\partial \Omega$.

The aim of the present paper is to show how can apply the theory of Dirichlet-to-Neumann operators on the localisation inverse problem in the framework of application outlined in problem (P2), which consists in finding the sufficient (Dirihlet-to-Neumann) conditions to localise the position of cell $\bar{B}$ from the experimentally measurable macroscopic response parameters.

In Section 2, we introduce the existence and uniqueness for the solution of problem (P2). In Section 3, we present our main results which consist in showing that total current (1.3), involving Dirihlet-to-Neumann operator (1.2), can resolve the localisation inverse problem in the two-dimensional case, when the compact $\Omega \subset \mathbb{R}^{2}$. We allow an explicit calculations for the solution of problem (P2) from Dirichlet-Neumann boundary conditions. Whereas for this solution, we use a method of conformal mapping for harmonic functions in doubly connected domains $\Omega \backslash B$.

\section{Uniqueness of the Problem (P2)}

We suppose that $\Omega$ and $B \subset \Omega$ be open bounded domains in $\mathbb{R}^{d}$ with $C^{2}$-smooth disjoint boundaries $\partial \Omega$ and $\partial B$, that is $\partial(\Omega \backslash \bar{B})=\partial \Omega \bigcup \partial B$ and $\partial \Omega \cap \partial B=\varnothing$.
Then the unit outer-normal to the boundary $\partial(\Omega \backslash \bar{B})$ vector-field $v(x), x \in \partial(\Omega \backslash \bar{B})$ is well-defined, and we consider the normal derivative in (P2) as the interior limit:

$$
\left.\left(\partial_{\nu} u\right)\right|_{\partial B}(\omega):=\lim _{x \rightarrow \omega} v(\omega) \cdot(\nabla u)(x), x \in \Omega \backslash \bar{B} .
$$

The existence of the limit (2.1) as well as the restriction $\left.u\right|_{\partial в}(\omega):=\lim _{x \rightarrow \omega} u(x)$ is insured since $u$ has to be harmonic solution of problem (P2) for $C^{2}$-smooth boundaries $\partial(\Omega \backslash \bar{B})$ [7].

Now, we introduce some indispensable standard notations and definitions, see [8]. Let $\mathcal{H}$ be Hilbert space $L^{2}(M)$ on domain $M \subset \mathbb{R}^{d}$ and $\partial \mathcal{H}:=L^{2}(\partial M)$ denote the corresponding boundary space. We denote by $W_{2}^{s}(M)$ the Sobolev space of $\mathcal{H}$-functions, whose $s$-derivatives are also in $\mathcal{H}$, and similar, $W_{2}^{s}(\partial M)$ is the Sobolev space of $\partial \mathcal{H}$-functions on the $C^{2}$-smooth boundary $\partial M$.

Proposition 2.1 Let $f \in W_{2}^{1 / 2}(\partial \Omega)$ for $C^{2}$-smooth boundaries $\partial(\Omega \backslash \bar{B})$. Then the Dirichlet-Neumann problem (P2) has a unique (harmonic) solution in domain $\Omega \backslash \bar{B}$.

Proof. For existence we refer to [7]. To prove the uniqueness, we consider the problem (P2) for $f=0$ and $c^{*}=0$. Then by Gauss-Ostrogradsky theorem, one gets that the corresponding solution $u$ yields:

$$
\begin{aligned}
& \int_{\Omega \backslash \bar{B}} \mathrm{~d} x(\nabla \overline{u(x)} \cdot \nabla u)(x) \\
& =\int_{\Omega \backslash \bar{B}} \mathrm{~d} x \operatorname{div}(\overline{u(x)}(\nabla u)(x)) \\
& =\int_{\partial B} \mathrm{~d} \sigma(\omega) \overline{u(\omega)}\left(\partial_{\nu} u\right)(\omega) \\
& =-W D^{-1} \int_{\partial B} \mathrm{~d} \sigma(\omega)|u(\omega)|^{2} \leq 0 .
\end{aligned}
$$

The estimate (2.2) implies that $u(x \in \Omega \backslash \bar{B})=$ Const . Hence by the boundary condition one gets

$\left.\left(W D^{-1} u\right)\right|_{\partial B}(\omega)=0$, and from $\left.u\right|_{\partial \Omega}(x)=f(x \in \partial \Omega)=0$, we obtain that for $W D^{-1} \geq 0$, the harmonic function $u(x)=0$ for $x \in \Omega \backslash \bar{B}$. $\square$

The next statement is a key for analysis of inverse localisation problems:

Proposition 2.2 Consider two problems (P2) corresponding to a bounded domain $\Omega \subset \mathbb{R}^{2}$ with $C^{2}$-smooth boundary $\partial \Omega$ and to two subsets $B_{1}$ and $B_{2}$ with the same smoothness of the boundaries $\partial B_{1}, \partial B_{2}$. If for solutions $u_{f}^{(1)}, u_{f}^{(2)}$ of these problems one has

$$
\left.\partial_{v} u_{f}^{(1)}\right|_{\partial \Omega}=\left.\partial_{v} u_{f}^{(2)}\right|_{\partial \Omega}
$$

then $\partial B_{1}=\partial B_{2}$. 
Proof. By virtue of $\left.u_{f}^{(1)}\right|_{\partial \Omega}=\left.u_{f}^{(2)}\right|_{\partial \Omega}=f$ and by condition (2.3), the problem (P2) has two solutions for identical external (on $\partial \Omega$ ) and internal (on $\partial B_{1}$ and $\partial B_{2}$ ) Robin boundary conditions. Then by the standard arguments based on the Holmgren uniqueness theorem [9] for harmonic functions on $\mathbb{R}^{2}$, one obtains that $\partial B_{1}=\partial B_{2}$.

\section{Inverse Problem: Conformal Mapping}

Let $\Omega$ and $B \subset \Omega$ be respectively open bounded domains in $\mathbb{R}^{2}$ with $C^{2}$-smooth disjoint boundaries $\partial \Omega=C\left(O_{0}, R_{0}\right)$ and $\partial B=C\left(O, r_{0}\right)$, where $C\left(O_{0}, R_{0}\right)$ and $C\left(O, r_{0}\right)$ are two circles respectively with radius $R_{0}$ and $r_{0}$.

In the sequel, we denote by $d_{0}$ the distance between the two centers $d_{0}=d_{O_{0} \rightarrow O}$.

The solution of the inverse localisation problem is decomposed into two steps:

In the first step, we introduce the necessary conformal mapping, see [10]. There are two reasons that make interest for using this technique, indeed the convenable conformal mapping $T$ :

(a) Transforms two non-concentric circles into two concentric circles.

(b) For any harmonic function $u, u \circ T$ still harmonic, see Proposition 3.1.

With this technique, we transform problem (P2) to another problem (P2)*, whose (P2)* has as solution an harmonic function also, and as domains $T(\Omega)$ and $T(B)$ which are concentric.

Therefore, we can find easily the general form for the solution of (P2)*, and consequently, we conclude the necessary coefficients for the general solution of problem (P2), with which we will be able to resolve its inverse problem.

In the next step, we are interested by resolving the localisation inverse problem using the explicit formula of $d_{0}$, which will be calculated in terms of (measurable) Dirichlet-Neumann boundary hypothesis on $\partial \Omega$.

Proposition 3.1 Let $T: N \rightarrow M$ be a conformal mapping defined by:

$$
z=\left(x_{1}, x_{2}\right) \rightarrow T(z):=\tilde{x}_{1}\left(x_{1}, x_{2}\right)+\mathrm{i} \tilde{x}_{2}\left(x_{1}, x_{2}\right) .
$$

If $u(x, y)$ is an harmonic function in $M$, then the composition

$$
\tilde{u}\left(\tilde{x}_{1}, \tilde{x}_{2}\right):=(u \circ T)\left(x_{1}, x_{2}\right)=u\left[x_{1}\left(\tilde{x}_{1}, \tilde{x}_{2}\right), x_{2}\left(\tilde{x}_{1}, \tilde{x}_{2}\right)\right],
$$

is an harmonic function in $N$.

In particular, by distinguishing explicitly the Laplacian in different coordinates, $\Delta_{z}:=\partial_{x_{1}}^{2}+\partial_{x_{2}}^{2}$ and $\Delta_{T}:=\partial_{\tilde{x}_{1}}^{2}+\partial_{\tilde{x}_{2}}^{2}$, one obtains:

$$
\Delta_{T} \tilde{u}\left[\tilde{x}\left(x_{1}, x_{2}\right), \tilde{y}\left(x_{1}, x_{2}\right)\right]=\left|\partial_{z} T(z)\right|^{-2} \Delta_{z} u\left(x_{1}, x_{2}\right) .
$$

\subsection{Necessary Conformal Mapping}

Let $T: \mathbb{C} \mapsto \mathbb{C}$ be the conformal mapping defined by: ${ }^{1}$

$$
\begin{aligned}
& T(z):=i(z+d i)(e z+i)^{-1} \\
& \text { that is }: T\left[z=\left(x_{1}, x_{2}\right)\right]=\tilde{x}_{1}+i \tilde{x}_{2},
\end{aligned}
$$

where:

$$
\begin{aligned}
& d=\frac{d_{0}^{2}+r_{0}^{2}-R_{0}^{2}+\sqrt{\left(d_{0}^{2}-r_{0}^{2}\right)^{2}+R_{0}^{4}-2 R_{0}^{2}\left(d_{0}^{2}+r_{0}^{2}\right)}}{2 d_{0}} \\
& \text { and } e=\frac{d}{r_{0}^{2}} .
\end{aligned}
$$

Remark 1 We define the conformal mapping $T$ relatively to the orthonormal reference with origin $O$ and axis $Y^{\prime} Y$, which is keen on the line $O O_{0}$ in the sense of the vector $\mathbf{O O}_{0}$.

Corollary 3.2 Ttransforms $C\left(O_{0}, R_{0}\right)$ and $C\left(O, r_{0}\right)$ from non-concentric circles to concentric circles $C\left(O, \tilde{R}_{0}\right)$ and $C\left(O, r_{0}\right)$, where $\tilde{R}_{0}$ is defined by:

$$
\tilde{R}_{0}=\sqrt{\frac{R_{0}^{2}+\left(d_{0}+d\right)^{2}}{\left(R_{0}^{2}+d_{0}^{2}\right) e^{2}+1+2 d_{0} e}} .
$$

Remark 2 Notice that when $d_{0} \rightarrow 0$, the mapping $T$ converges to the identity function.

\subsection{Problem (P2)*}

Let $F$ and $\tilde{f}$ be respectively the values of the normal derivative $\left.\partial_{\nu} C\right|_{\partial в}$ and $f$ with the new variables $\left(\tilde{x}_{1}, \tilde{x}_{2}\right)$, i.e:

$$
\left\{\begin{array}{l}
F\left(\tilde{x}_{1}, \tilde{x}_{2}\right):=\left.\partial_{\nu} C\left(x_{1}, x_{2}\right)\right|_{\partial B}, \\
\tilde{f}\left(\tilde{x}_{1}, \tilde{x}_{2}\right):=f\left(x_{1}, x_{2}\right) .
\end{array}\right.
$$

Then, if we make the substitution $C\left(x_{1}, x_{2}\right)=\tilde{C}\left(\tilde{x}_{1}, \tilde{x}_{2}\right)$, then by using the conformal mapping given in (3.1), we show from proposition 3.1 that the problem (P2) can be transformed to problem (P2)*, which obeys the set of equations:

$$
\text { (P2)* }^{*}\left\{\begin{array}{l}
\Delta \tilde{C}\left(\tilde{x}_{1}, \tilde{x}_{2}\right)=0 \\
\left(\tilde{x}_{1}, \tilde{x}_{2}\right) \in D\left(O, \tilde{R}_{0}\right) \backslash D\left(O, r_{0}\right), \\
\tilde{C}\left(\tilde{x}_{1}, \tilde{x}_{2}\right)=\tilde{f}\left(\tilde{x}_{1}, \tilde{x}_{2}\right) \\
\text { on } C\left(O, \tilde{R}_{0}\right) \\
-D F\left(\tilde{x}_{1}, \tilde{x}_{2}\right)=W\left[\tilde{C}\left(\tilde{x}_{1}, \tilde{x}_{2}\right)-c^{*}\right] \quad \text { on } C\left(O, r_{0}\right)
\end{array}\right.
$$

${ }^{1} \mathrm{i}$ is the complex number. 
In the sequel, we denote by $\tilde{\rho}$ and $\tilde{\varphi}$ the polar coordinates associated to the variables $\left(\tilde{x}_{1}, \tilde{x}_{2}\right)$.

Corollary 3.3 The solution of the problem (P2)* gets the form:

$$
\begin{aligned}
\tilde{C}(\tilde{\rho}, \tilde{\varphi}) & =\tilde{\alpha}+\tilde{\beta} \ln \tilde{\rho} \\
& +\sum_{k=1}^{+\infty}\left[\tilde{c}_{1}(k) \tilde{\rho}^{k}+\tilde{c}_{2}(k) \tilde{\rho}^{-k}\right] \cos (k \tilde{\varphi}) \\
& +\left[\tilde{c}_{3}(k) \tilde{\rho}^{k}+\tilde{c}_{4}(k) \tilde{\rho}^{-k}\right] \sin (k \tilde{\varphi}) .
\end{aligned}
$$

Proof. As $\tilde{C}$ is harmonic, then the proof follows, see [10]. $\square$

Hereinafter, we need to make explicit $F$ in order to calculate the coefficients of the development (3.4).

Proposition 3.4 The function $F$ is given by:

$$
\begin{aligned}
& \mathrm{F}(\tilde{\rho}, \tilde{\varphi})=\frac{1}{r_{0}(1-e d)} \\
& \times\left\{(1+e d) \tilde{\beta}-\left(e r_{0}+d r_{0}^{-1}\right) \tilde{\beta} \sin \tilde{\varphi}\right. \\
& +(1+e d) \sum_{k=1}^{+\infty} k\left[r_{0}^{k} \tilde{c}_{1}(k)-r_{0}^{-k} \tilde{c}_{2}(k)\right] \cos (k \tilde{\varphi}) \\
& \quad+k\left[r_{0}^{k} \tilde{c}_{3}(k)-r_{0}^{-k} \tilde{c}_{4}(k)\right] \sin (k \tilde{\varphi}) \\
& +\sum_{k=1}^{+\infty} \frac{k}{2}\left[r_{0}^{k-1} \tilde{c}_{1}(k)-r_{0}^{-k-1} \tilde{c}_{2}(k)\right][\sin (k+1) \tilde{\varphi}-\sin (k-1) \tilde{\varphi}] \\
& +\frac{k}{2}\left[r_{0}^{k-1} \tilde{c}_{3}(k)-r_{0}^{-k-1} \tilde{c}_{4}(k)\right] \\
& \quad[\cos (k-1) \tilde{\varphi}-\cos (k+1) \tilde{\varphi}]\}
\end{aligned}
$$

Proof. Since $F\left(\tilde{x}_{1}, \tilde{x}_{2}\right):=\left.\partial_{v} C\left(x_{1}, x_{2}\right)\right|_{\partial B}$, we deduce from the definition of $\left.\partial_{v} C\left(x_{1}, x_{2}\right)\right|_{\partial B}$ that:

$$
\begin{aligned}
F\left(\tilde{x}_{1}, \tilde{x}_{2}\right) & :=\partial_{\nu} C\left(x_{1}, x_{2}\right) \\
& :=r_{0}^{-1}\left(x_{1} \partial_{x_{1}} C+x_{2} \partial_{x_{2}} C\right) .
\end{aligned}
$$

We need firstly to calculate $\partial_{x_{1}} C$ and $\partial_{x_{2}} C$ in terms of $\left(\tilde{x}_{1}, \tilde{x}_{2}\right)$. By virtue of the substitution $C\left(x_{1}, x_{2}\right)=\tilde{C}\left(\tilde{x}_{1}, \tilde{x}_{2}\right)$, we obtain the following differential relation:

$$
\begin{aligned}
\mathrm{d} C\left(x_{1}, x_{2}\right) & =\mathrm{d} \tilde{C}\left(\tilde{x}_{1}, \tilde{x}_{2}\right) \\
& =\partial_{\tilde{x}_{1}} \tilde{C}\left(\tilde{x}_{1}, \tilde{x}_{2}\right) \mathrm{d} \tilde{x}_{1}+\partial_{\tilde{x}_{2}} \tilde{C}\left(\tilde{x}_{1}, \tilde{x}_{2}\right) \mathrm{d} \tilde{x}_{2} .
\end{aligned}
$$

Then by comparison, we conclude that:

$$
\begin{aligned}
& \partial_{x_{1}} C=\partial_{\tilde{x}_{1}} \tilde{C}\left(\tilde{x}_{1}, \tilde{x}_{2}\right) \partial_{x_{1}} \tilde{x}_{1}+\partial_{\tilde{x}_{2}} \tilde{C}\left(\tilde{x}_{1}, \tilde{x}_{2}\right) \partial_{x_{1}} \tilde{x}_{2}, \\
& \partial_{x_{2}} C=\partial_{\tilde{x}_{1}} \tilde{C}\left(\tilde{x}_{1}, \tilde{x}_{2}\right) \partial_{x_{2}} \tilde{x}_{1}+\partial_{\tilde{x}_{2}} \tilde{C}\left(\tilde{x}_{1}, \tilde{x}_{2}\right) \partial_{x_{2}} \tilde{x}_{2} .
\end{aligned}
$$

Substitute Equation (3.7) in Equation (3.6), one obtains:

$$
\begin{aligned}
& F\left(\tilde{x}_{1}, \tilde{x}_{2}\right) \\
& =r_{0}^{-1}\left[\left(x_{1} \partial_{x_{1}} \tilde{x}_{1}+x_{2} \partial_{x_{2}} \tilde{x}_{1}\right) \partial_{\tilde{x}_{1}} \tilde{C}+\left(x_{1} \partial_{x_{1}} \tilde{x}_{2}+x_{2} \partial_{x_{2}} \tilde{x}_{2}\right) \partial_{\tilde{x}_{2}} \tilde{C}\right]
\end{aligned}
$$

Recall that Equation (3.1) gives us a relation between the variables $\left(x_{1}, x_{2}\right)$ and $\left(\tilde{x}_{1}, \tilde{x}_{2}\right)$. Then, the quantities $\left(x_{1} \partial_{x_{1}} \tilde{x}_{1}+x_{2} \partial_{x_{2}} \tilde{x}_{1}\right)$ and $\left(x_{1} \partial_{x_{1}} \tilde{x}_{2}+x_{2} \partial_{x_{2}} \tilde{x}_{2}\right)$ in Equation (3.8) can be calculated in terms of $\left(\tilde{x}_{1}, \tilde{x}_{2}\right)$. Therefore we have:

$$
\begin{aligned}
& F\left(\tilde{x}_{1}, \tilde{x}_{2}\right)=\frac{1}{r_{0}(1-e d)} \\
& \cdot\left\{\tilde{x}_{1}\left(1+e d-2 e \tilde{x}_{2}\right) \partial_{\tilde{x}_{1}} C\right. \\
& \left.\quad+\left[e\left(\tilde{x}_{1}^{2}-\tilde{x}_{2}^{2}\right)+(1+e d) \tilde{x}_{2}-d\right] \partial_{\tilde{x}_{2}} \tilde{C}\right\}
\end{aligned}
$$

Finally, if we replace $\tilde{x}_{1}$ and $\tilde{x}_{2}$ by their associated polar coordinates $(\tilde{\rho}, \tilde{\varphi})$, then we can rewrite $\mathrm{F}\left(\tilde{x}_{1}, \tilde{x}_{2}\right)$ in terms of $\tilde{\rho}$ and $\tilde{\varphi}$ (due to the relation between polar derivatives and cartesian derivatives) as follows:

$$
\begin{aligned}
& \mathrm{F}(\tilde{\rho}, \tilde{\varphi})=\frac{1}{r_{0}(1-e d)} \\
& \cdot\left[(1+e d) r_{0} \partial_{\tilde{\rho}} \tilde{C}(\tilde{\rho}, \tilde{\varphi})-\left(e r_{0}^{2}+d\right) \sin \tilde{\varphi} \partial_{\tilde{\rho}} \tilde{C}(\tilde{\rho}, \tilde{\varphi})\right] .
\end{aligned}
$$

Therefore, it is enough to replace $\tilde{C}(\tilde{\rho}, \tilde{\varphi})$ by its value given in Equation (3.4). $\square$

In order to resolve problem (P2)*, we need to make explicit $\tilde{f}$. So, the change of variables given in Equation (3.1), allows us to express $\tilde{f}$ with the following Fourier series:

$$
\begin{aligned}
\tilde{f}\left(\tilde{x}_{1}, \tilde{x}_{2}\right)= & \alpha^{\tilde{f}} \\
& +\sum_{k=1}^{+\infty} c_{1}^{\tilde{f}}(k) \cos (k \tilde{\varphi})+c_{2}^{\tilde{f}}(k) \sin (k \tilde{\varphi}),
\end{aligned}
$$

where:

$$
\left\{\begin{aligned}
\alpha^{\tilde{f}} & =\frac{1}{2 \pi} \int_{0}^{2 \pi} \mathrm{d} \tilde{\varphi} \tilde{f}\left(\tilde{x}_{1}, \tilde{x}_{2}\right)=\frac{1}{2 \pi} \int_{0}^{2 \pi} \mathrm{d} \tilde{\varphi}\left(x_{1}, x_{2}\right) f\left(x_{1}, x_{2}\right), \\
c_{1}^{\tilde{f}}(k) & =\frac{1}{\pi} \int_{0}^{2 \pi} \mathrm{d} \tilde{\varphi} \tilde{f}\left(\tilde{x}_{1}, \tilde{x}_{2}\right) \cos (k \tilde{\varphi}) \\
& =\frac{1}{\pi} \int_{0}^{2 \pi} \mathrm{d} \tilde{\varphi}\left(x_{1}, x_{2}\right) f\left(x_{1}, x_{2}\right) \cos \left[k \tilde{\varphi}\left(x_{1}, x_{2}\right)\right], \\
c_{2}^{\tilde{f}}(k) & =\frac{1}{\pi} \int_{0}^{2 \pi} \mathrm{d} \tilde{\varphi} \tilde{f}\left(\tilde{x}_{1}, \tilde{x}_{2}\right) \sin (k \tilde{\varphi}) \\
& =\frac{1}{\pi} \int_{0}^{2 \pi} \mathrm{d} \tilde{\varphi}\left(x_{1}, x_{2}\right) f\left(x_{1}, x_{2}\right) \sin \left[k \tilde{\varphi} f\left(x_{1}, x_{2}\right)\right] .
\end{aligned}\right.
$$

Remark 3 Notice that the coefficients of (16) depend only of: 
1. $\tilde{\varphi}\left(x_{1}, x_{2}\right)$ given from the change of variables due to $(3.1)$.

2. $f$ which is the external condition boundary of problem (P2).

Proposition 3.5 The coefficients of Equation (3.4) are given by:

$$
\tilde{\alpha}=\alpha^{\tilde{f}}-\tilde{\beta} \ln \tilde{R}_{0} \text { and } \tilde{\beta}=\frac{Q_{1}}{Q_{2}}, \text { whose : }
$$

$$
\begin{aligned}
& Q_{1}=-2 W D^{-1} r_{0}(1-e d)\left(\alpha^{\tilde{f}}-c^{*}\right)+\tilde{R}_{0} r_{0}^{-2} c_{2}^{\tilde{f}}(1)+\frac{(1-e d)(1+e d)^{-1} D^{-1} \tilde{R}_{0} W\left(1+\tilde{R}_{0}^{2} r_{0}^{-2}\right) c_{2}^{\tilde{f}}(1)}{r_{0}+\tilde{R}_{0}^{2} r_{0}^{-1}+D^{-1} W r_{0}(1+e d)^{-1}(1-e d)\left(r_{0}-\tilde{R}_{0}^{2} r_{0}^{-1}\right)-\tilde{R}_{0} r_{0}^{-1} c_{2}^{\tilde{f}}(1)}, \\
& Q_{2}=2(1+e d)+2 D^{-1} r_{0}(1-e d) W \ln \left(r_{0} \tilde{R}_{0}^{-1}\right)+\frac{\left(e r_{0}+d r_{0}^{-1}\right)(1+e d)^{-1}\left(1+\tilde{R}_{0}^{2} r_{0}^{-2}\right)}{r_{0}+\tilde{R}_{0}^{2} r_{0}^{-1}+D^{-1} W r_{0}(1-e d)(1+e d)^{-1}\left(r_{0}-\tilde{R}_{0}^{2} r_{0}^{-1}\right)-\tilde{R}_{0} r_{0}^{-1} c_{2}^{\tilde{f}}(1)} .
\end{aligned}
$$

Proof. First boundary condition of problem (P2)* implies: $\left.\tilde{C}\right|_{C\left(o, \tilde{R}_{0}\right)}\left(\tilde{x}_{1}, \tilde{x}_{2}\right)=\tilde{f}\left(\tilde{x}_{1}, \tilde{x}_{2}\right)$. Then, by replacing $\tilde{C}\left(\tilde{x}_{1}, \tilde{x}_{2}\right)$ and $\tilde{f}\left(\tilde{x}_{1}, \tilde{x}_{2}\right)$ by their values given respectively in (3.4) and (3.10), we obtain after identification that:

$$
\left\{\begin{array}{l}
\tilde{\alpha}+\tilde{\beta} \ln \tilde{R}_{0}=\alpha \tilde{f} \\
\text { identification between the constants, } \\
\tilde{c}_{2}(1)+\tilde{c}_{1}(1) \tilde{R}_{0}^{2}=c_{1}^{\tilde{f}}(1) \tilde{R}_{0} \\
\text { identification between the coefficients of } \cos (\tilde{\varphi}), \\
\tilde{c}_{4}(1)+\tilde{c}_{3}(1) \tilde{R}_{0}^{2}=c_{2}^{\tilde{f}}(1) \tilde{R}_{0} \\
\text { identification between the coefficients of } \sin (\tilde{\varphi}) .
\end{array}\right.
$$

Afterwards, from the second boundary condition in problem (P2)*, we have

$-D F(\tilde{\rho}, \tilde{\varphi})=W\left[\left.\tilde{C}\right|_{C\left(O, r_{0}\right)}(\tilde{\rho}, \tilde{\varphi})-c^{*}\right]$. Then, by the similar manner, we deduce from (3.5) and (3.4) that:

$$
\left\{\begin{array}{l}
(1+e d) \tilde{\beta}+\frac{1}{2}\left[\tilde{c}_{3}(1)-r_{0}^{-2} \tilde{c}_{4}(1)\right] \\
=-D^{-1} r_{0}(1-e d) W\left(\tilde{\alpha}+\tilde{\beta} \ln r_{0}-c^{*}\right) \\
(1+e d)\left[\tilde{c}_{1}(1) r_{0}-r_{0}^{-1} \tilde{c}_{2}(1)\right] \\
=-D^{-1} r_{0}(1-e d) W\left[\tilde{c}_{1}(1) r_{0}+r_{0}^{-1} \tilde{c}_{2}(1)\right] \\
\left(e r_{0}^{2}+d\right) \tilde{\beta}+(1+e d)\left[r_{0}^{2} \tilde{c}_{3}(1)-\tilde{c}_{4}(1)\right] \\
=D^{-1} r_{0}(1-e d) W\left[r_{0}^{2} \tilde{c}_{3}(1)+\tilde{c}_{4}(1)\right]
\end{array}\right.
$$

Finally, one has a system of six equations with six unknowns $\tilde{\alpha}, \tilde{\beta}, \tilde{c}_{1}(1), \tilde{c}_{2}(1), \tilde{c}_{3}(1)$ and $\tilde{c}_{4}(1)$. The solution of this system ends the proof.

Remark 4 Notice that the proof of Proposition 3.5 show us the advantage of conformal mapping technique. Indeed, the identification between Fourier series on the boundary conditions of problem (P2)* is easily calculated because its boundaries are two concentric circles, and consequently its radius are constant. But, it is not the case in problem (P2), because here its boundaries are non-concentric, whose its radius depend of polar angle.

Remark 5 For the inverse problem, we will just need the explicit value of $\tilde{\alpha}$ and $\tilde{\beta}$, that why we didn't make explicit the other coefficients values for the Fourier series of $\tilde{C}$.

\subsection{Localisation Inverse Problem}

For resolving the inverse problem, we need the following:

(i) First, we aim to calculate the total flux $J_{\partial \Omega}$ across the external boundary $\partial \Omega$. For that, we need to express the solution $C$ of problem (P2) in terms of Fourier series.

(ii) Second, we aim to find an equation for $d_{0}$.

In the sequel, we denote by $\rho$ and $\varphi$ the polar coordinates associated to the initial variables $\left(x_{1}, x_{2}\right)$.

(i) The solution of problem (P2) gets the following form:

$$
\begin{aligned}
& C(\rho, \varphi)=\alpha+\beta \ln \rho \\
& +\sum_{k=1}^{+\infty}\left[c_{1}(k) \rho^{k}+c_{2}(k) \rho^{-k}\right] \cos (k \varphi) \\
& \quad+\left[c_{3}(k) \rho^{k}+c_{4}(k) \rho^{-k}\right] \sin (k \varphi)
\end{aligned}
$$

where $\alpha, \beta, c_{1}(k), c_{2}(k), c_{3}(k)$ and $c_{4}(k)$ for all $k \in \mathbb{N}$, are the Fourier coefficients of $C$.

Corollary 3.6 The total flux $J_{\partial \Omega}$ and $J_{\partial B}$ satisfy the following:

$$
J_{\partial \Omega}=J_{\partial B}=-2 \pi D \beta
$$

Proof. Since the differential element $\mathrm{d} l$ at boundary $\partial B$ are respectively equal to $r_{0} \mathrm{~d} \varphi$, then by inserting (3.12) in (1.2), we deduce that:

$$
J_{\partial B}:=\int_{M \in \partial B} \mathrm{~d} l \boldsymbol{j}_{M} \cdot \boldsymbol{n}_{M}=-2 \pi D \beta,
$$

where $\boldsymbol{j}_{M}:=-D \nabla C(M)$ and $\boldsymbol{n}_{M}=\boldsymbol{e}_{r}$ designed respectively local current and outer-normal vector at arbitrary point $M$.

On the other hand, by Gauss-Ostrogradsky theorem, one gets: 


$$
\begin{aligned}
& J_{\partial \Omega}-J_{\partial B}:=\int_{M \in \partial \Omega \bigcup \partial B} \mathrm{~d} l \boldsymbol{j}_{M} \cdot \boldsymbol{n} \\
& =-D \int_{\Omega \backslash \bar{B}} \mathrm{~d} S \nabla \cdot \nabla C=-D \int_{\Omega \backslash \bar{B}} \mathrm{~d} S \Delta C=0,
\end{aligned}
$$

where $\mathrm{d} S$ is the areal differential element. Therefore, (19) is deduced. $\square$

(ii) We now state the following corollary and proposition which will be important to find a relation between the coefficients $\tilde{\alpha}$ and $\tilde{\beta}$ involving $d_{0}$.

\section{Corollary 3.7}

$$
-D \beta=r_{0} W\left(\alpha+\beta \ln r_{0}-c^{*}\right) .
$$

Proof. Substitute $C(\rho, \varphi)$ in the second boundary condition of problem (P2) (at the interface $\partial B$ of cell $\bar{B})$ by its value given in Equation (3.12), therefore the proof follows by identification (between two Fourier series). $\square$

\section{Proposition 3.8}

$$
\alpha+\beta \ln r_{0}=\tilde{\alpha}+\tilde{\beta} \ln r_{0} .
$$

Proof. Since $C\left(x_{1}, x_{2}\right)=\tilde{C}\left(\tilde{x}_{1}, \tilde{x}_{2}\right)$, one gets also $C(\rho, \varphi)=\tilde{C}(\tilde{\rho}, \tilde{\varphi})$. But, we have $T\left[\partial B=C\left(O, r_{0}\right)\right]=C\left(O, r_{0}\right)$, then $C\left(r_{0}, \varphi\right)=\tilde{C}\left(r_{0}, \tilde{\varphi}\right)$.

On the other hand, $(\varphi, \tilde{\varphi})$ varies in $[0,2 \pi[\times[0,2 \pi[$. So, by applying the double integral on the domain $\left[0,2 \pi\left[\times\left[0,2 \pi\left[\right.\right.\right.\right.$ for the equality $C\left(r_{0}, \varphi\right)=\tilde{C}\left(r_{0}, \tilde{\varphi}\right)$, and by replacing $C\left(r_{0}, \varphi\right)$ and $\tilde{C}\left(r_{0}, \tilde{\varphi}\right)$ by their values given respectively in Equations (3.4) and (3.12), we deduce that:

$$
\begin{aligned}
& \int_{0}^{2 \pi} \int_{0}^{2 \pi} \alpha+\beta \ln r_{0}+\sum_{k=1}^{+\infty}\left[r_{0}^{k} c_{1}(k)+r_{0}^{-k} c_{2}(k)\right] \cos (k \varphi)+\left[r_{0}^{k} c_{3}(k)+r_{0}^{-k} c_{4}(k)\right] \sin (k \varphi) \mathrm{d} \varphi \mathrm{d} \tilde{\varphi} \\
& =\int_{0}^{2 \pi} \int_{0}^{2 \pi} \tilde{\alpha}+\tilde{\beta} \ln r_{0}+\sum_{k=1}^{+\infty}\left[r_{0}^{k} \tilde{c}_{1}(k)+r_{0}^{-k} \tilde{c}_{2}(k)\right] \cos (k \tilde{\varphi})+\left[r_{0}^{k} \tilde{c}_{3}(k)+r_{0}^{-k} \tilde{c}_{4}(k)\right] \sin (k \tilde{\varphi}) \mathrm{d} \varphi \mathrm{d} \tilde{\varphi} .
\end{aligned}
$$

Then Equation (3.15) follows from Fubini's theorem.

Finally, we aim to find the equation satisfied by $d_{0}$.

In fact, by inserting (3.13) and (3.14) in Equation (3.15), we obtain:

$$
r_{0}^{-1} W^{-1} J_{\partial \Omega}+2 \pi c^{*}-=2 \pi\left(\tilde{\alpha}+\tilde{\beta} \ln r_{0}\right) .
$$

\section{Conclusions}

From Proposition 3.5, we remark that $\tilde{\alpha}$ and $\tilde{\beta}$ are calculated in terms of $e, d$ and $\tilde{R}_{0}$, which their formulae given in (3.2) and (3.3) depend of $d_{0}$ and the coefficients of (3.10).

Then, Equation (3.16) becomes an equation of the only unknown $d_{0}$ involving the parameters $J_{\partial \Omega}$ (1.3) and $f:=\left.C\right|_{\partial \Omega}$ (see Remark 3), which are the Dirichlet-toNeumann hypothesis of problem (P2) on the external boundary, and we can found them from an experimental measures.

To summarize, we have found an equation for $d_{0}$, which is the distance between the center $O$ of cell $\bar{B}$ and the center $O_{0}$ of $\Omega$, so it remains to find the position of the center $O$. In fact:

Let $M_{\max }$ and $M_{\min }$ be two points at the external boundary $\partial \Omega$ whose the norm of the local current $\boldsymbol{j}$ reaches respectively its maximum and minimum values, see Figure 1. Then, from the symmetry of the shape, we deduce that the center $O$ of cell $\bar{B}$ is localized at the line passed by the points $M_{\max }, M_{\min }$ and $O_{0}$, exactly between $M_{\max }$ and $O_{0}$ where the distance $d_{0}$ be- tween $O$ and $O_{0}$ is given by Equation (3.16).

By conclusion, we can now answer the question posed in the introduction about the uniqueness of the inverse localisation problem associated to (P2), and we can conclude that the total flux (1.3) is sufficient to resolve the localisation inverse problem, in two-dimensional case, if the shape is regular. But, it is not enough in other type of inverse problem like geometrical inverse problem, see [11].

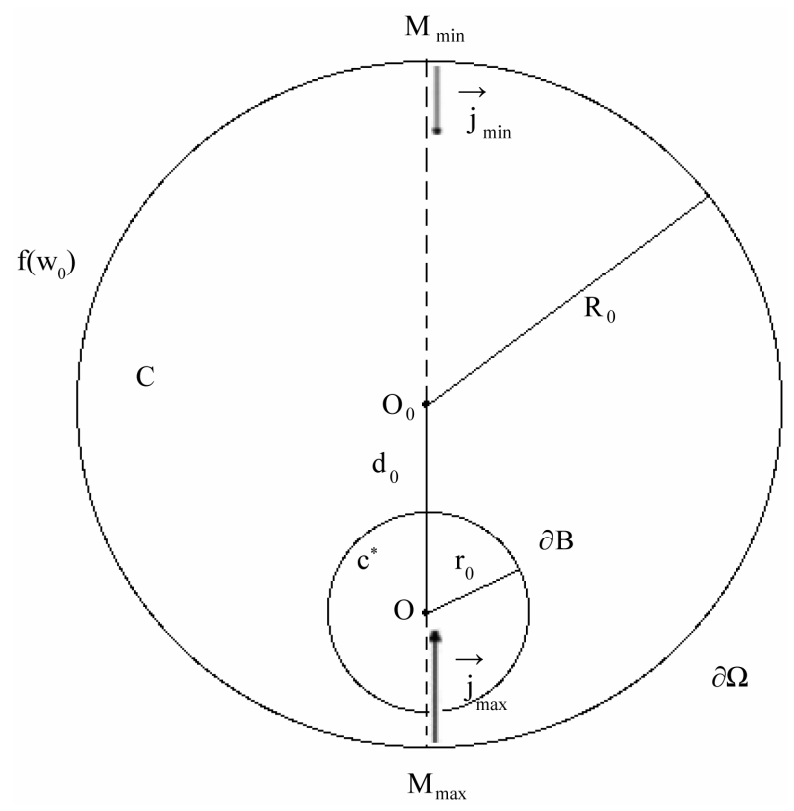

Figure 1. Position of cell $\bar{B}$. 


\section{Acknowledgements}

I want to thank the CNRS Federation "Francilienne de Mecanique, Materiaux Structures et Procedes" CNRS FR2609, in particularly Prof. Didier Clouteau, for financial support in this study.

\section{REFERENCES}

[1] J. Lee and G. Uhlmann, Communications on Pure and Applied Mathematics, Vol. 42, 1989, pp. 1097-1112. doi:10.1002/cpa.3160420804

[2] D. C. Barber and B. H. Brown, Journal of Physics E: Scientific Instruments, Vol. 17, 1984, pp. 723-733. doi:10.1088/0022-3735/17/9/002

[3] M. E. Taylor, "Pseudodifferential Operators," Princeton University Press, Princeton, 1996.

[4] A. Greenleaf and G. Uhlmann, Duke Mathematical Journal, Vol. 108, 2001, pp. 599-617. doi:10.1215/S0012-7094-01-10837-5
[5] B. Sapoval, Physical Review Letters, Vol. 73, 1994, pp. 3314-3316. doi:10.1103/PhysRevLett.73.3314

[6] D. S. Grebenkov, M. Filoche and B. Sapoval, Physical Review E, Vol. 73, 2006, Article ID: 021103. doi:10.1103/PhysRevE.73.021103

[7] M. E. Taylor, "Partial Differential Equations II: Qualitative Studies of Linear Equations," Springer-Verlag, Berlin, 1996. doi:10.1007/978-1-4757-4187-2

[8] J. K. Hunter and B. Nachtergaele, "Applied Analysis," World Scientific, Singapore City, 2001.

[9] D. Tataru, Communications in Partial Differential Equations, Vol. 20, 1995, pp. 855-884.

[10] I. Baydoun, “Opérateurs de Dirichlet-Neumann et Leurs Applications: Transport Laplacien, Problème Inverse et Opérateur de Dirichlet-Neumann," Éditions Universitaires Européennes, 2012.

[11] I. Baydoun and V. A. Zagrebnov, Theoretical and Mathematical Physics, Vol. 168, 2011, pp. 1180-1191. doi:10.1007/s11232-011-0097-8 